\title{
A mathematical model for cell infiltration and proliferation in a chondral defect
}

\author{
L.S. Kimpton ${ }^{\mathrm{a}}$, A. Schwab ${ }^{\mathrm{b}}$, F. Ehlicke ${ }^{\mathrm{b}}$, S.L. Waters ${ }^{\mathrm{a}}$, C.P. Please ${ }^{\mathrm{a}}$, \\ J.P. Whiteley ${ }^{\mathrm{c}, *}$, H.M. Byrne ${ }^{\mathrm{a}}$ \\ ${ }^{a}$ Mathematical Institute, University of Oxford, Andrew Wiles Building, Radcliffe \\ Observatory Quarter, Woodstock Road, Oxford OX2 6GG, United Kingdom \\ ${ }^{b}$ University Hospital Wuerzburg, Department of Tissue Engineering and Regenerative \\ Medicine (TERM), Roentgenring 11, 97070 Wuerzburg, Germany \\ ${ }^{c}$ Department of Computer Science, University of Oxford, Wolfson Building, Parks Road, \\ Oxford OX1 3QD, United Kingdom
}

\begin{abstract}
We develop a mathematical model to describe the regeneration of a hydrogel inserted into an ex vivo osteochondral explant. Specifically we use partial differential equations to describe the evolution of two populations of cells that migrate from the tissue surrounding the defect, proliferate, and compete for space and resources within the hydrogel. The two cell populations are chondrocytes and cells that infiltrate from the subchondral bone. Model simulations are used to investigate how different seeding strategies and growth factor placement within the hydrogel affect the spatial distribution of both cell types. Since chondrocyte migration is extremely slow, we conclude that the hydrogel should be seeded with chondrocytes prior to culture in order to obtain zonal chondrocyte distributions typical of those associated with healthy cartilage.
\end{abstract}

\footnotetext{
*Corresponding author

Email address: jonathan.whiteley@cs.ox.ac.uk (J.P. Whiteley)
} 
Keywords: cartilage, hydrogel, osteochondral, tissue engineering

\section{Introduction}

When cartilage is damaged, through injury or disease, it has very little capacity for self-repair. Tissue engineering has become a popular approach for treating damaged cartilage, the goal being either to engineer new cartilage tissue that can be transplanted into a defect or to enhance the reparative capacity of the damaged cartilage [1]. Experimental techniques include the insertion of hydrogels, either with or without cells, into a cartilage defect. The performance of such engineered constructs will be improved by better understanding how cartilage growth and repair are regulated by different hydrogels, different cell seeding strategies and/or different growth factors. In healthy cartilage, chondrocytes are found in high concentrations in the superficial layer, at the surface of the cartilage, and in lower concentrations deeper in the cartilage, towards the bone $[2,3,4]$. A key goal for the progress of tissue engineered cartilage treatments is to engineer tissue that mimics this layered zonal architecture seen in native cartilage [5].

An experimental osteochondral defect model has recently been developed to investigate cartilage regeneration treatments. Briefly, porcine osteochondral biopsies are isolated and a full-thickness cartilage defect (4mm diameter) is created. The biopsies are cultured ex vivo under physiological conditions in a custom made culture platform. The experimental model allows the testing of different treatment strategies in a complex 3D environment. Possible defect treatment approaches are either with cell free or cell loaded scaffold materials. We focus on one class of scaffold materials, namely hydrogels. In 


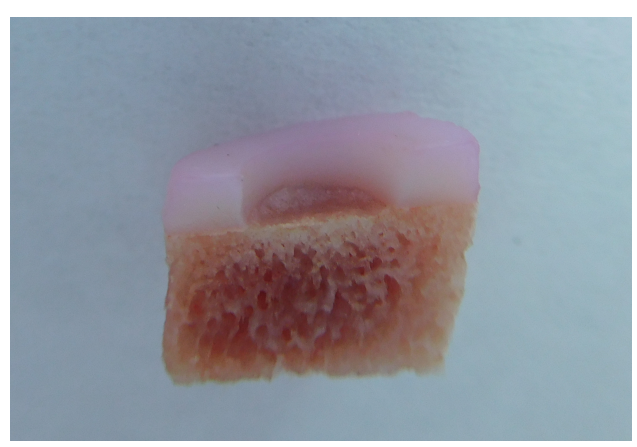

(a)

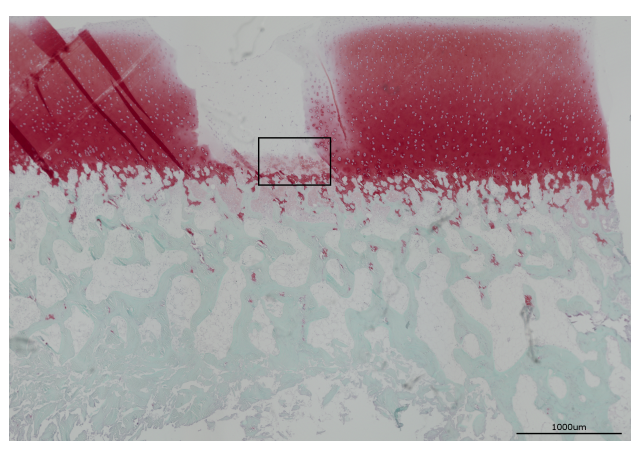

(c)

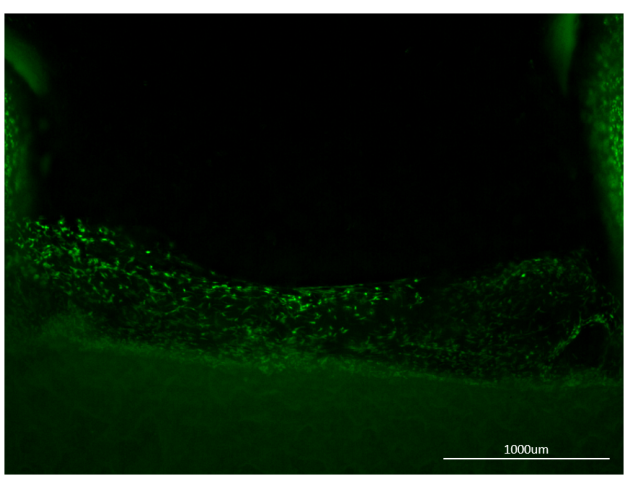

(b)

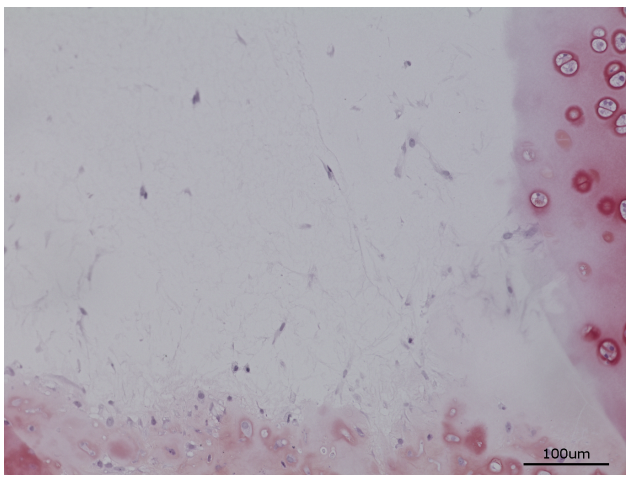

(d)

Figure 1: Porcine osteochondral explant with cartilage defect and cross section of explant with cell free hydrogel treatment after 28 days of ex vivo culture. (a) Macroscopic view of a freshly isolated osteochondral explant with $4 \mathrm{~mm}$ full thickness cartilage defect. (b) Calcein staining of metabolic active cells in osteochondral explant with cell free hydrogel after ex vivo culture. Cross section shows living cells (marked in green) that migrated from surrounding tissue into the hydrogel. Scale bar is $1000 \mu \mathrm{m}$. (c) Safranin-O staining of osteochondral explant cross section with cartilage defect. Proteoglycans in cartilage matrix are stained in red and subchondral bone in light blue. Scale bar is $1000 \mu \mathrm{m}$. (d) Higher magnification of safranin-O staining (boxed region in (c)). During ex vivo culture cells (cell nuclei are marked in black) from the surrounding tissue migrated into the hydrogel producing cartilage-like tissue at the cartilage-bone interface. Scale bar is $100 \mu \mathrm{m}$. 
the literature different attempts to enhance cartilage defect regeneration have been discussed, either by modifying hydrogel materials with growth factors like TGF- $\beta$, or investigating cell seeding strategies $[6,7,8]$.

Alongside the hydrogels being tested in the osteochondral model there are additional factors influencing cartilage regeneration. The surrounding cartilage and bone tissue of the experimental model provides a natural cell source involved in tissue regeneration, one that has been successfully utilised in healing rabbit cartilage defects [9]. The first cell population, originating from the surrounding cartilage tissue, are chondrocytes. The second cell population comprises all cells from the subchondral bone potentially including osteoblasts, osteoclasts, mesenchymal stem cells or hematopoietic cells (from now on referred to as bone cells). Mature chondrocytes in healthy cartilage are thought to undergo little proliferation or migration. The proliferative capacity of chondrocytes (or chondrocyte-like cells) in different hydrogels is less clear and authors have presented evidence that growth factors, used individually or in combination, enhance chondrocyte proliferation; these include TGF- $\beta[6,10]$ or a combination of TGF- $\beta$, FGF-2 and PDGF-BB [11]. The ability of mature chondrocytes to migrate also remains contentious $[12,13]$ and, whilst it is likely that chondrocyte migration is very slow, there is evidence that certain growth factors, for example rhBMP-2 [14] and bFGF [15], enhance chondrocyte migration. There is also evidence that mononuclear cells derived from the peripheral blood enhance chondrocyte migration [13] and that fibrin sealant can increase both chondrocyte migration and proliferation [16].

To illustrate these points, in Figure 1(a) we present experimental images 
from a cross section of a freshly isolated porcine osteochondral explant with full thickness cartilage defect. To study the migration of cells from the surrounding cartilage and bone tissue of the osteochondral biopsies, the defects were treated with a cell free hydrogel (Fibrin glue, Baxter) and cultured for 28 days. To determine cell viability half of the biopsies were incubated with $4 \mathrm{mM}$ calcein AM (Life Technologies) and visualized with fluorescence microscope (Keyence BZ-9000, Biorevo). Metabolically active, elongated cells that migrated from the subchondral bone into the cell free hydrogel were stained green (Figure 1(b)). They were localized in the bottom of the hydrogel filled defect, next to the calcified layer, the interface between bone and cartilage. For histological stainings biopsies were fixed with $4 \%$ Roti $^{\circledR}$ Histofix (Carl Roth), plastic embedded (T9100, Heraeus Kulzer), deplastified, rehydrated and stained with Safranin-O (0.1\% v/v, Sigma Aldrich) and Fast Green (0.01\% v/v, Sigma Aldrich). Cell nuclei were counterstained in black with Weigert Iron Hematoxylin (Morphisto). Proteoglycans in the cartilage were visualized in red and proteins in light blue. Safranin-O staining confirmed findings of cell invasion from subchondral bone and cartilage into the hydrogel, shown in Figure 1(d). Additionally, cartilage like matrix production was indicated by the light red staining of the hydrogel in the defect. Few cells have migrated and those that have are localised in the bottom of the hydrogel. Whether these cells originated from the cartilage or the bone, or whether they are a mixed population is not clear.

There is a plethora of choices in experimental design when attempting to tissue engineer cartilage. For example, if the hydrogel is cultured inside an ex vivo osteochondral explant, we may seed the hydrogel with cells, or 
we may rely on cell infiltration from the surrounding tissue. Further, if the hydrogel is seeded with cells, we must decide what type of cells to use and at what density they should be seeded. Given the observation that healthy cartilage tissue is characterized by the zonal chondrocyte distribution it is natural to ask if this layered tissue structure can be achieved in vitro by an appropriate choice of experimental design. With this in mind, non-uniform cell seeding strategies - with different cell types and/or different cell concentrations in different layers - represent a promising approach. Furthermore, it is of interest whether hydrogel material modifications in terms of attaching growth factors in different layers to create a gradient in concentration may be a beneficial strategy. An exhaustive search of all feasible combinations of experimental design would be costly and time-consuming. To facilitate the rapid comparison of different experiment designs we develop a simple mathematical model, with attention focussing on two cell populations that can migrate into the hydrogel from the cartilage and the bone. Our theoretical framework is presented in $\S 2$. In $\S 3$ we present results generated from numerical simulations of the model and use these both to interpret the images in Figure 1 and to suggest possible seeding strategies for engineering zonal cartilage tissue. Finally we discuss the conclusions of our work in $\S 4$.

\section{Model formulation}

We formulate a mathematical model to describe the migration and proliferation of cells in a chondral defect that has been filled with a hydrogel. We consider a cylindrical, full-thickness chondral defect as shown schematically in Figure 2. The model is formulated on the cylindrical domain occupied by 


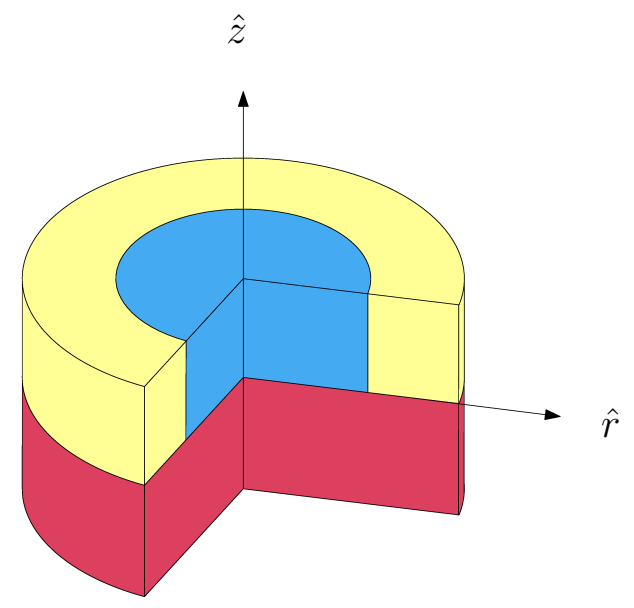

Figure 2: Schematic diagram defining the problem domain which is the hydrogel cylinder (blue region); for completeness, the surrounding cartilage (yellow region) and bone (red region) are also indicated. The hydrogel is positioned in a defect in the cartilage of an $e x$ vivo osteochondral explant.

the hydrogel; the effects of the surrounding bone and cartilage are incorporated into the model via boundary conditions.

We consider dimensional variables, denoted by hats, and exploit the geometry of the model by seeking axisymmetric solutions in a cylinder of height $\hat{L}$ and radius $\hat{R}$, with radial co-ordinate $0<\hat{r}<\hat{R}$ and depth co-ordinate $0<\hat{z}<\hat{L}$. We shall seek solutions in the time interval $0<\hat{t}<\hat{T}$. We suppose that there are two cell populations, those from the chondrocyte lineage (subsequently termed chondrocytes for brevity) with concentration $\hat{c}(\hat{r}, \hat{z}, \hat{t})$, and cells migrating in from the subchondral bone with concentration $\hat{b}(\hat{r}, \hat{z}, \hat{t})$ (both populations measured as cell volume per unit volume of hydrogel). Cells migrating in from the bone originate from the marrow, stroma or derive from the hematopoietic lineage including mesenchymal stem cells (MSCs), osteoblast and osteoclast precursor cells. Throughout this pa- 
per we use the term 'bone cells' to mean any combination of these cell types. We assume that migration and proliferation are the dominant processes affecting the two cell types and, hence, model their time evolution by the equations

$$
\begin{gathered}
\frac{\partial \hat{c}}{\partial \hat{t}}=\underbrace{\hat{\nabla} \cdot\left(-\hat{\mathbf{J}}_{c}\right)}_{\text {migration }}+\underbrace{\hat{\mu}_{c} \hat{c}\left(1-\frac{\hat{c}+\hat{b}}{\hat{M}}\right)}_{\text {proliferation }}, \\
\frac{\partial \hat{b}}{\partial \hat{t}}=\underbrace{\hat{\nabla} \cdot\left(-\hat{\mathbf{J}}_{b}\right)}_{\text {migration }}+\underbrace{\hat{\mu}_{b} \hat{b}\left(1-\frac{\hat{c}+\hat{b}}{\hat{M}}\right)}_{\text {proliferation }},
\end{gathered}
$$

where the cell fluxes $\hat{\mathbf{J}}_{c}$ and $\hat{\mathbf{J}}_{b}$ are given by

$$
\hat{\mathbf{J}}_{c}=-\frac{\hat{D}_{c}}{1+\hat{\alpha}_{c}(\hat{b}+\hat{c})} \hat{\nabla} \hat{c}, \quad \hat{\mathbf{J}}_{b}=-\frac{\hat{D}_{b}}{1+\hat{\alpha}_{b}(\hat{b}+\hat{c})} \hat{\nabla} \hat{b},
$$

in the region $0<\hat{r}<\hat{R}$ and $0<\hat{z}<\hat{L}$. We note that a range of approaches to modelling cell migration and proliferation with reaction-diffusion equations can be found in the literature $[17,18,19]$; for an introduction to the mathematical techniques used, see Edelstein-Keshet [20]. Here the rate of proliferation of both cell populations is assumed to reduce as the total cell concentration approaches the carrying capacity of the hydrogel and we denote by $\hat{\mu}_{i}(i=c, b)$ the maximum proliferation rates of the cell populations and $\hat{M}$ the carrying capacity of the hydrogel. In what follows, as here, we shall assume that the subscript $i$ can take values $c$ and $b$ for relevant parts of the two cell populations. We denote by $\hat{D}_{i}$ the constant diffusion coefficients of the two cell types and account for reductions in random cell motion due to crowding at high cell concentrations by introducing the constant pos- 
itive parameters $\hat{\alpha}_{i}$ to represent the degree of inhibition that the total cell population, $\hat{b}+\hat{c}$, has on the migration of each cell population.

To solve the coupled reaction-diffusion equations for $\hat{c}$ and $\hat{b}$ we must introduce suitable boundary and initial conditions. We assume that at $\hat{t}=0$ the spatial distributions of both cell types within the cylindrical hydrogel are known so that

$$
\hat{c}(\hat{r}, \hat{z}, 0)=\hat{c}_{0}(\hat{r}, \hat{z}), \quad \hat{b}(\hat{r}, \hat{z}, 0)=\hat{b}_{0}(\hat{r}, \hat{z})
$$

When imposing the boundary conditions, we suppose that no cells leave via the top surface of the hydrogel $(\hat{z}=\hat{L})$ and that the system is symmetric about $\hat{r}=0$. Thus, the flux of both cell populations may be set to zero on $\hat{z}=\hat{L}$ and on $\hat{r}=0$ and we have

$$
\begin{aligned}
\frac{\partial \hat{c}}{\partial \hat{z}}(\hat{r}, \hat{L}, \hat{t})=0, & \frac{\partial \hat{b}}{\partial \hat{z}}(\hat{r}, \hat{L}, \hat{t})=0, \\
\frac{\partial \hat{c}}{\partial \hat{r}}(0, \hat{z}, \hat{t})=0, & \frac{\partial \hat{b}}{\partial \hat{r}}(0, \hat{z}, \hat{t})=0 .
\end{aligned}
$$

Finally the boundary conditions on $\hat{z}=0$ and $\hat{r}=\hat{R}$ describe how the hydrogel interacts with the surrounding bone $(\hat{z}=0)$ and cartilage $(\hat{r}=\hat{R})$ and, in particular, the rate at which cells infiltrate into, or migrate out of, the hydrogel. At $\hat{r}=\hat{R}$ we impose

$$
\begin{aligned}
\hat{\mathbf{J}}_{b} \cdot \mathbf{e}_{r} & =-\frac{\hat{D}_{b}}{1+\hat{\alpha}_{b}(\hat{b}+\hat{c})} \frac{\partial \hat{b}}{\partial \hat{r}}=\hat{\Gamma}_{c} \hat{b}, \\
\hat{\mathbf{J}}_{c} \cdot \mathbf{e}_{r} & =-\frac{\hat{D}_{c}}{1+\hat{\alpha}_{c}(\hat{b}+\hat{c})} \frac{\partial \hat{c}}{\partial \hat{r}}=-\hat{\Gamma}_{c}\left(k_{1} \hat{C}-\hat{c}\right),
\end{aligned}
$$


and at $\hat{z}=0$ we impose

$$
\begin{aligned}
-\hat{\mathbf{J}}_{b} \cdot \mathbf{e}_{z} & =\frac{\hat{D}_{b}}{1+\hat{\alpha}_{b}(\hat{b}+\hat{c})} \frac{\partial \hat{b}}{\partial \hat{z}}=-\hat{\Gamma}_{b}\left(k_{2} \hat{B}-\hat{b}\right), \\
-\hat{\mathbf{J}}_{c} \cdot \mathbf{e}_{z} & =\frac{\hat{D}_{c}}{1+\hat{\alpha}_{c}(\hat{b}+\hat{c})} \frac{\partial \hat{c}}{\partial \hat{z}}=\hat{\Gamma}_{b} \hat{c} .
\end{aligned}
$$

where $\mathbf{e}_{r}, \mathbf{e}_{z}$ are unit vectors in the increasing $\hat{r}$ and $\hat{z}$ directions, respectively. In these boundary conditions $\hat{B}$ and $\hat{C}$ denote the concentrations of the two cell types in the bone and the cartilage respectively, and the parameters $\hat{\Gamma}_{i}(i=c, b)$ quantify the rates at which cells cross the bone-hydrogel and cartilage-hydrogel interfaces. The parameters $k_{1}$ and $k_{2}$ are constants of proportionality, that indicate the relative affinity of the cells for the hydrogel compared to the bone or the cartilage. If $k_{2}>1$ then bone cells prefer to be in the hydrogel than in the subchondral bone, whereas if $k_{2}<1$ bone cells prefer to be in the bone than the hydrogel. For example, if $k_{2}=2$ then net migration of bone cells will be from the bone into the hydrogel whilst the concentration of bone cells in the hydrogel at $\hat{z}=0$ is less than twice the concentration of bone cells in the neighbouring bone. A similar interpretation applies to the constant $k_{1}$ that appears in Eq. (8) relating to the influx of cells at the interface between the cartilage and the hydrogel.

\subsection{Parameter estimates}

Before continuing, it is convenient to rewrite the governing equations in nondimensional form; see [21] for details on this mathematical technique. In order to do so we require estimates of values of the parameters that appear in the governing equations. A typical defect has dimensions $\hat{R}=2 \mathrm{~mm}$ and $\hat{L}=2 \mathrm{~mm}$. Following [19], we estimate the chondrocyte proliferation rate to 
be $\hat{\mu}_{c} \approx 4.8 \times 10^{-3} \mathrm{day}^{-1}$ and fix $\hat{\mu}_{b} \approx 0.13 \mathrm{day}^{-1}$ as a typical proliferation rate for osteoblasts [22]. The diffusion coefficient for chondrocytes is assumed to lie in the range $\hat{D}_{c}=10^{-3}-1.5 \times 10^{-3} \mathrm{~mm}^{2}$ day $^{-1}[23,24]$. We estimate a typical bone cell diffusion coefficient of $\hat{D}_{b}=0.1 \mathrm{~mm}^{2} \mathrm{day}^{-1}[22]$. In the absence of literature estimates for the remaining model parameters, we use values that yield qualitative agreement between numerical simulations and our preliminary experimental data, i.e. cell concentrations in the hydrogel are low and the maximum cell concentrations are found along the base of the hydrogel. In more detail, we fix $\hat{\alpha}_{c}=\hat{\alpha}_{b}=\hat{M}, k_{1} \hat{C}=k_{2} \hat{B}=\hat{M}$, $\hat{\Gamma}_{c}=0.01 / 14 \mathrm{~mm} \mathrm{day}^{-1}$ and $\hat{\Gamma}_{b}=0.1 / 14 \mathrm{~mm} \mathrm{day}^{-1}$.

\subsection{Nondimensional equations}

We use the typical radius of the hydrogel $\hat{R}=2 \mathrm{~mm}$, the duration of the

experiment $\hat{T}=28$ days, and a typical carrying capacity $\hat{M}$ to nondimensionalise the governing equations by setting

$$
\hat{r}=\hat{R} r, \quad \hat{z}=\hat{R} z, \quad \hat{t}=\hat{T} t, \quad \hat{c}=\hat{M} c, \quad \hat{b}=\hat{M} b
$$

If we also introduce the following dimensionless parameter groupings $D_{i}=$ $\hat{D}_{i} T / L^{2}, \mu_{i}=\hat{\mu}_{i} T, \alpha_{i}=\hat{\alpha}_{i} \hat{M}, \Gamma_{i}=\hat{\Gamma}_{i} T / L, C=k_{1} \hat{C} / \hat{M}, B=k_{2} \hat{B} / \hat{M}$ and $L=\hat{L} / \hat{R}$ then the governing equations for $c$ and $b$ can be written

$$
\begin{aligned}
& \frac{\partial c}{\partial t}=\nabla \cdot\left(\frac{D_{c}}{1+\alpha_{c}(b+c)} \nabla c\right)+\mu_{c} c(1-c-b), \\
& \frac{\partial b}{\partial t}=\nabla \cdot\left(\frac{D_{b}}{1+\alpha_{b}(b+c)} \nabla b\right)+\mu_{b} b(1-c-b),
\end{aligned}
$$

with initial and boundary conditions given by

$$
c(r, z, 0)=c_{0}(r, z), \quad b(r, z, 0)=b_{0}(r, z)
$$




$$
\begin{array}{llrl}
\frac{\partial c}{\partial z}(r, L, t) & =0, & & \frac{\partial b}{\partial z}(r, L, t)=0 \\
\frac{\partial c}{\partial r}(0, z, t) & =0, & \frac{\partial b}{\partial r}(0, z, t) & =0
\end{array}
$$

and

$$
\frac{D_{b}}{1+\alpha_{b}(b+c)} \frac{\partial b}{\partial r}=-\Gamma_{c} b, \quad \frac{D_{c}}{1+\alpha_{c}(b+c)} \frac{\partial c}{\partial r}=\Gamma_{c}(C-c),
$$

at $r=1$ and

$$
\frac{D_{b}}{1+\alpha_{b}(b+c)} \frac{\partial b}{\partial z}=-\Gamma_{b}(B-b), \quad \frac{D_{c}}{1+\alpha_{c}(b+c)} \frac{\partial c}{\partial z}=\Gamma_{b} c,
$$

at $z=0$.

\section{Results}

The governing equations (12)-(18) are solved numerically using the finite element method; see [25] for more details. Mesh refinement studies were carried out (not shown) to ensure that further mesh refinement does not significantly alter the results. We shall present the results of simulations for unseeded hydrogels and investigate the effect of varying the proliferation rates and interface permeabilities on cell infiltration of the hydrogel. We shall also model a hydrogel with zonal enhancement of chondrocyte proliferation and migration and consider different hydrogel seeding strategies. We conclude by modelling the healing response to a wound, where the quantities $B$ and $C$ that appear in Eqs. (17) and (18) are functions of time.

\subsection{Unseeded, spatially-uniform hydrogels}

We solve the governing equations for a hydrogel which is cell-free so that $c_{0}(r, z)=b_{0}(r, z)=0$ in Eq. (14). Unless otherwise stated, we use, as default, the nondimensional parameter values in Table 1. 


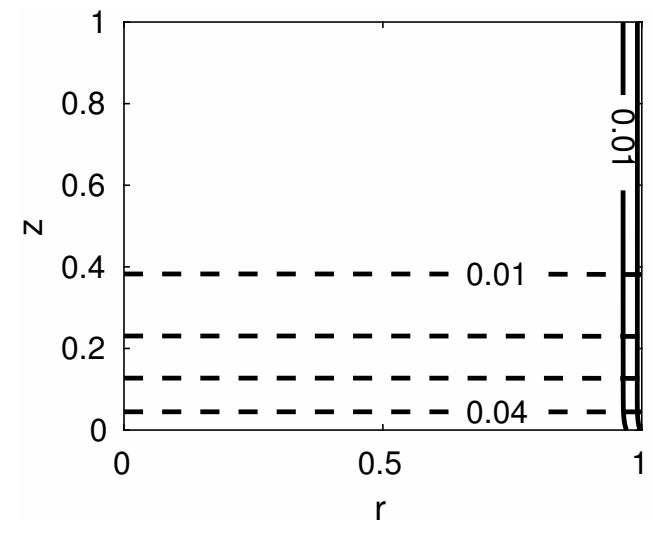

(a) $t=0.1$

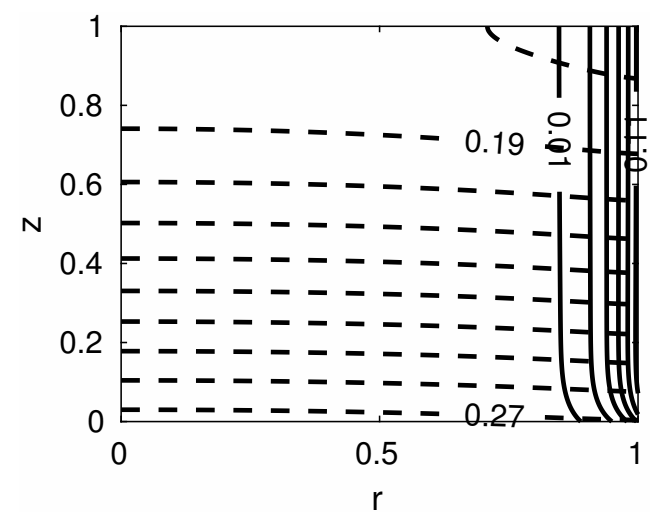

(c) $t=0.7$

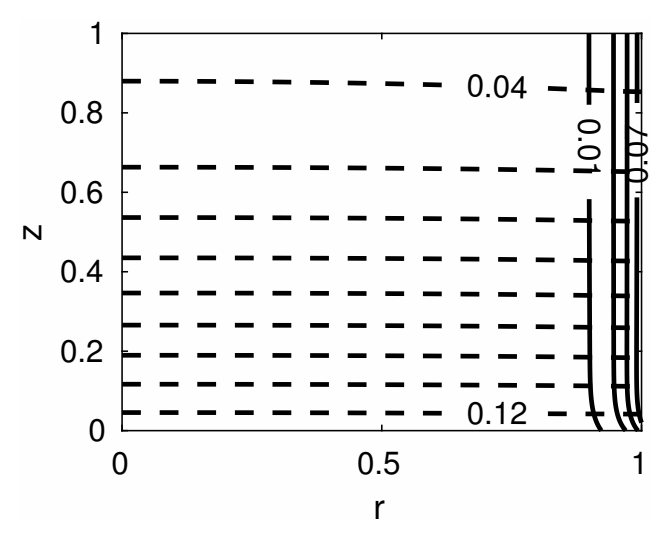

(b) $t=0.4$

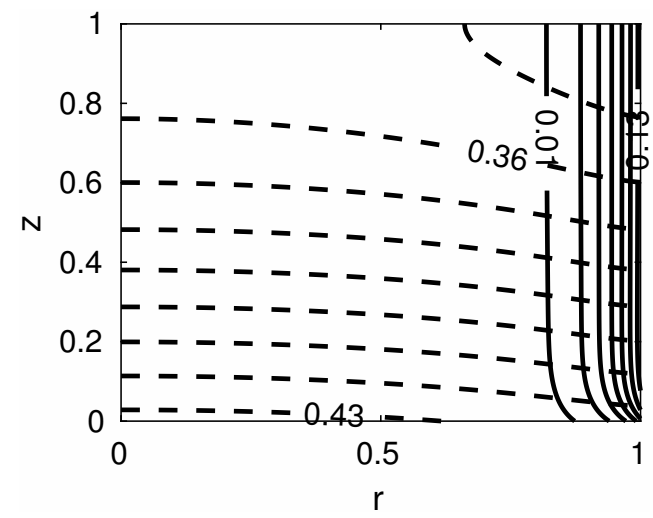

(d) $t=1$

Figure 3: The solution of Eqs. (12)-(18) using parameter values given in Table 1. Contour plots of the chondrocyte concentrations (solid lines) and bone cell concentrations (broken lines) for an initially unseeded hydrogel $\left(c_{0}=b_{0}=0\right)$ reveal more rapid and extensive cell infiltration by bone cells from the subchondral bone $(z=0)$ than chondrocytes from the cartilage $(r=1)$. Panels (a), (b), (c) and (d) show the cell concentrations at times $t=0.1,0.4,0.7$ and 1 (which are equivalent to 2.8, 11.2, 19.6 and 28 days of culture. The maximum chondrocyte concentration in panel (a) is 0.03 . 
Table 1: List of typical nondimensional parameter values

\begin{tabular}{ll}
\hline Parameter & Value \\
\hline$L=\hat{L} / \hat{R}$ & 1 \\
$D_{c}=\hat{D}_{c} T / L^{2}$ & 0.008 \\
$D_{b}=\hat{D}_{b} T / L^{2}$ & 0.7 \\
$\mu_{b}=\hat{\mu}_{b} T$ & 3 \\
$\mu_{c}=\hat{\mu}_{c} T$ & 0.1 \\
$\alpha_{b}=\hat{\alpha_{b}} \hat{M}$ & 1 \\
$\alpha_{c}=\hat{\alpha_{c}} \hat{M}$ & 1 \\
$\Gamma_{c}=\hat{\Gamma_{c}} T / L$ & 0.01 \\
$\Gamma_{b}=\hat{\Gamma_{b}} T / L$ & 0.1 \\
$C=k_{1} \hat{C} / \hat{M}$ & 1 \\
$B=k_{2} \hat{B} / \hat{M}$ & 1 \\
\hline
\end{tabular}

In Figure 3 we plot contours of both the chondrocyte and bone cell concentration at times $t=0.1, t=0.4, t=0.7$ and $t=1$, which correspond to $2.8,11.2,19.6$ and 28 days of culture. (In all contour plots the bone contours are plotted at 0.01 intervals and the chondrocyte contours at 0.02 intervals, unless otherwise stated.) The figure shows the concentration of bone cells is highest near the hydrogel-bone interface and decreases towards the top of the hydrogel, and that the bone cell concentrations exceed those of the chondrocytes, with this difference becoming more pronounced over time. We observe a small population of chondrocytes localised near to the hydrogel-cartilage interface, a distribution which is not representative of that associated with healthy cartilage.

The model provides additional information to that which is obtainable 


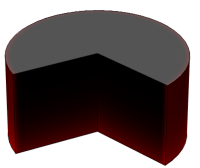

(a) $t=0.1$

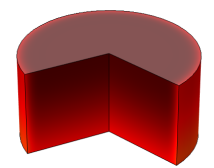

(b) $t=0.4$

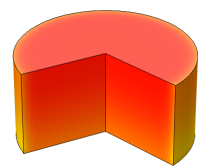

(c) $t=0.7$

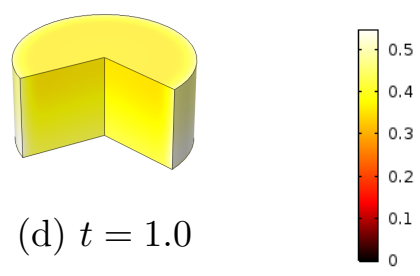

(d) $t=1.0$

Figure 4: Series of plots showing how the total cell concentration $(b+c)$ within an initially unseeded hydrogel $\left(c_{0}=b_{0}=0\right)$ changes over time. Parameter values as per Figure 3 (see Table 1).

experimentally, because it allows us to easily distinguish between the two cell types. For comparison with experimental observations we must sum $b$ and $c$ and plot the total cell concentration. In Figure 4 we show how the combined cell concentration, $(b+c)$, in the hydrogel increases as time progresses. Additionally, at each time point the cell concentration near to the hydrogel-bone interface and the hydrogel-cartilage interface exceeds that at the top and in the centre of the hydrogel.

Uncertainty in the parameter values leads us to consider the effect that variations in their values has on the two cell populations. In Figure 5 we show how the cell concentration contours change when the cell proliferation rates vary, (i.e. we consider large and small values of $\hat{\mu}_{b}, \hat{\mu}_{c}$ while holding all other parameters at the control values used in Figure 3). We observe that a ten-fold reduction in the chondrocyte proliferation rate (from $\mu_{c}=0.1$ to $\left.\mu_{c}=0.01\right)$ has a negligible effect on the bone cell and chondrocyte contours (see Figures 3(d) and 5(a)). By contrast, a fifty-fold increase in $\mu_{c}$ (from $\mu_{c}=0.1$ to $\left.\mu_{c}=5\right)$ dramatically increases the chondrocyte concentration close to the hydrogel-cartilage interface, (see Figures 3(d) and 5(b)) but the 
chondrocytes remain confined within a narrow band of the hydrogel, close to $r=1$, where the bone cell concentration is reduced due to competition with the chondrocytes. Varying the bone cell proliferation rate $\mu_{b}$ has little effect on the chondrocytes (see Figures 5(c) and 5(d)). At very large values of $\mu_{b}$ the bone cell concentration is close to one throughout the hydrogel; in dimensional terms, this corresponds to the bone cell concentration being close to the carrying capacity, $\hat{M}$. There is still some variation in the bone cell concentration across the hydrogel; it reduces near to $r=1$ due to migration of bone cells out of the hydrogel, into the surrounding cartilage. Even with large changes in the proliferation rates we are unable to replicate qualitatively distributions of chondrocytes that vary with vertical depth into the explant and/or reproduce distributions similar to those that characterise healthy cartilage.

To generate a summary statistic that allows us to directly compare the extent to which each cell population colonises the hydrogel we average $c$ and $b$ over the domain $0<r<1,0<z<L$. We report the average nondimensional cell concentrations in Figure 6 for a range of proliferation constants. We see that increasing $\mu_{c}$ yields a modest increase in the chondrocyte content, with little effect on the bone cells. Even for large values of $\mu_{c}$ the average chondrocyte concentration achieved by $t=1$ (corresponding to 28 days) is small. We note also that increasing $\mu_{b}$ increases the bone cell content, but has little effect on the chondrocyte content.

In Figure 7 we display the chondrocyte and bone cell profiles that result from changing the interface permeabilities $\Gamma_{c}$ and $\Gamma_{b}$, all other parameters being held fixed at their control values. As expected, for small $\Gamma_{c}$ 


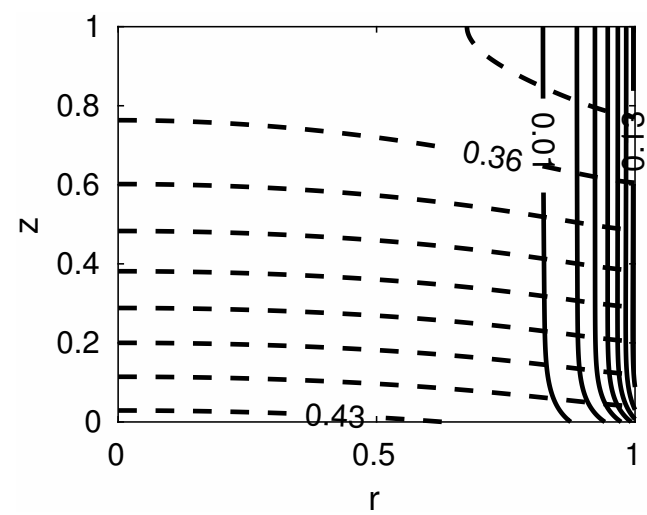

(a) $\mu_{c}=0.01, \mu_{b}=3$

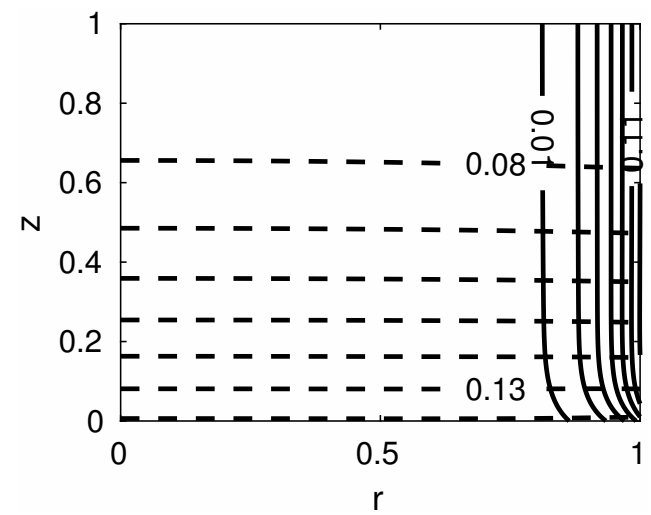

(c) $\mu_{c}=0.1, \mu_{b}=0.1$

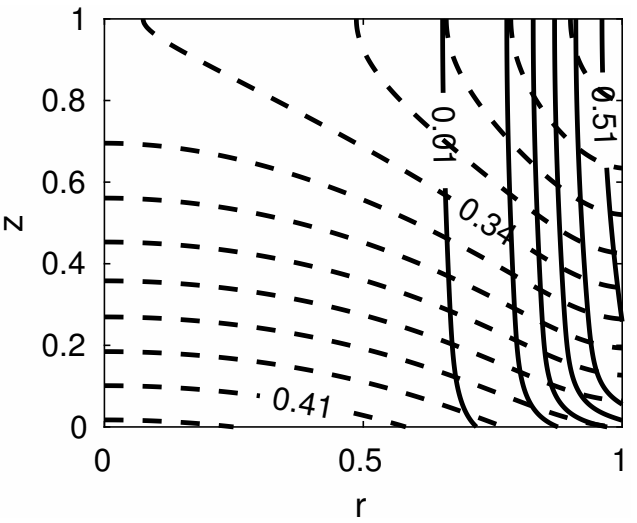

(b) $\mu_{c}=5, \mu_{b}=3$

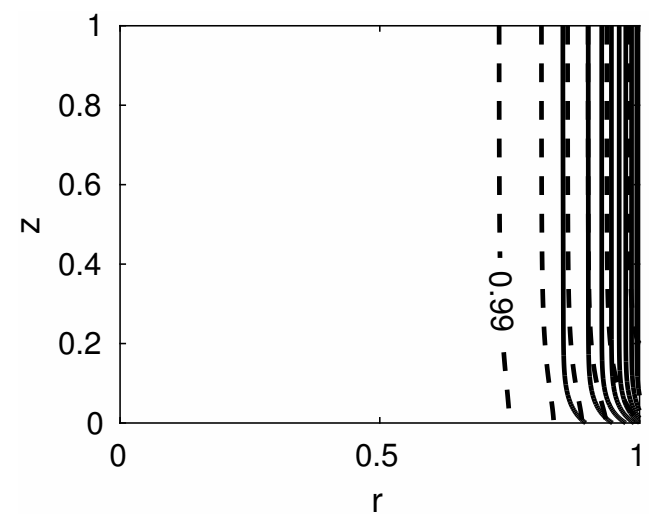

(d) $\mu_{c}=0.1, \mu_{b}=30$

Figure 5: Series of plots showing how varying the cell proliferation rates $\mu_{c}$ and $\mu_{b}$ affects the distribution of chondrocytes (solid lines) and bone cells (broken lines) at $t=1$ with hydrogels that are initially devoid of cells $\left(c_{0}(r, z)=b_{0}(r, z) \equiv 0\right)$. All parameter values, with the exception of the proliferation constants $\mu_{c}$ and $\mu_{b}$, are held at the fixed values stated in Table 1. In (a) $\mu_{c}=0.01$ and $\mu_{b}=3$. In (b) $\mu_{c}=5$ and $\mu_{b}=3$, and the chondrocyte contours are at intervals of 0.1. In (c) $\mu_{c}=0.1$ and $\mu_{b}=0.1$. In (d) $\mu_{c}=0.1$, $\mu_{b}=30$, the chondrocyte contours shown are between 0.01 and 0.15 (highest values near to $r=1$ ) and the bone contours shown are between 0.99 and 0.94 . 


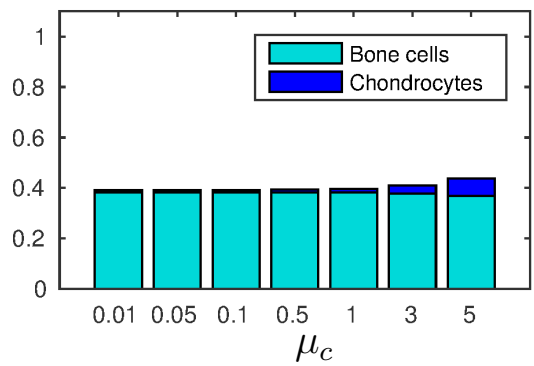

(a)

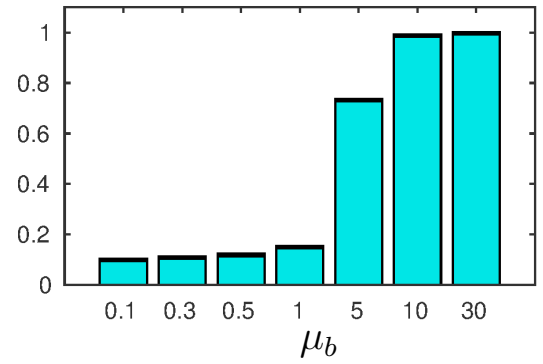

(b)

Figure 6: Seris of bar plots showing how the average bone cell and chondrocyte content at $t=1$ change as the cell proliferation rates $\mu_{c}$ and $\mu_{b}$ vary. These simulations were computed for a hydrogel that was initially devoid of cells. Parameter values: as per Figure 5, except that in (a) $\mu_{b}=3$ and in (b) $\mu_{c}=0.1$.

values the chondrocyte concentration is reduced because it is more difficult for chondrocytes to migrate into the hydrogel (see Figure 7(a)). Increasing the permeability of the hydrogel-cartilage interface increases the chondrocyte concentration, but the chondrocyte population remains localised near the cartilage (see Figure $7(\mathrm{~b})$ ). Increasing $\Gamma_{c}$ also reduces bone cell levels near to the cartilage as it is easier for the bone cells to migrate out of the hydrogel and there is increased competition for resources from the elevated chondrocyte numbers. Varying the permeability of the hydrogel-bone interface, $\Gamma_{b}$ has little impact on the number or distribution of chondrocyte numbers (see Figures 7(c) and 7(d)). Smaller values of $\Gamma_{b}$ restrict the number of bone cells migrating into the hydrogel. We note that it is not possible to replicate the layered chondrocyte concentration observed experimentally by varying the permeabilities.

In Figure 8 we show how average cell numbers at time $t=1$ (correspond- 


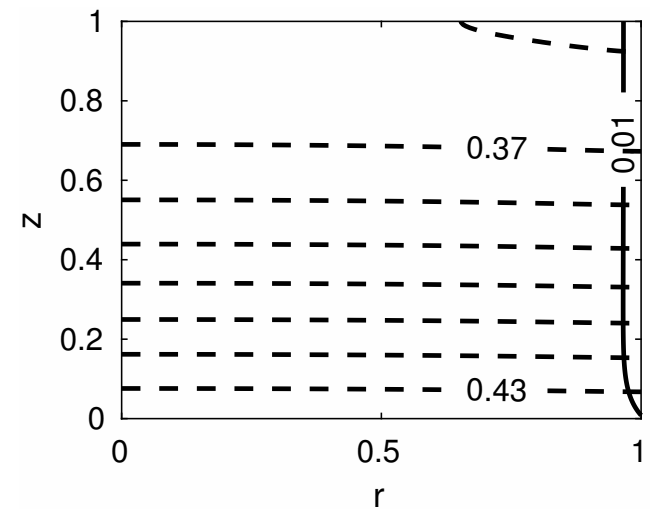

(a) $\Gamma_{c}=0.001, \Gamma_{b}=0.1$

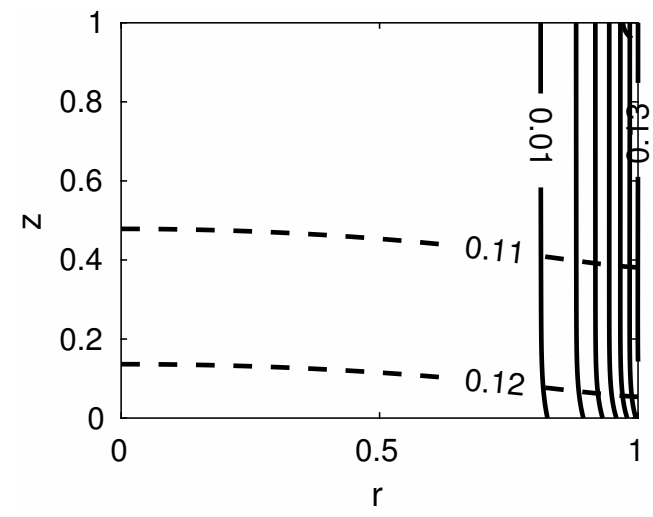

(c) $\Gamma_{c}=0.01, \Gamma_{b}=0.02$

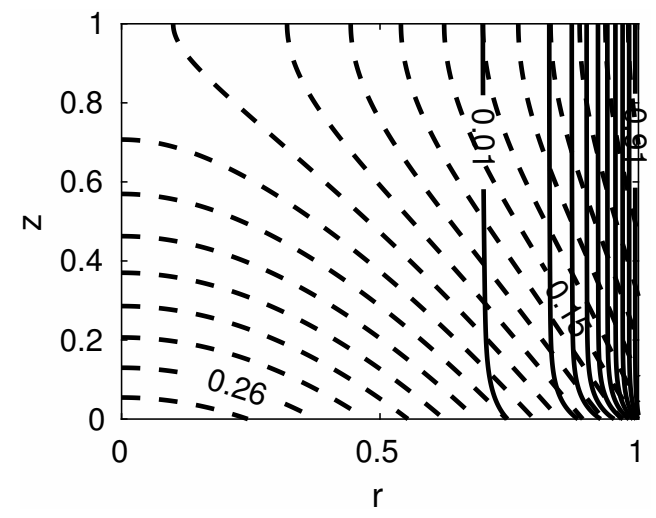

(b) $\Gamma_{c}=1, \Gamma_{b}=0.1$

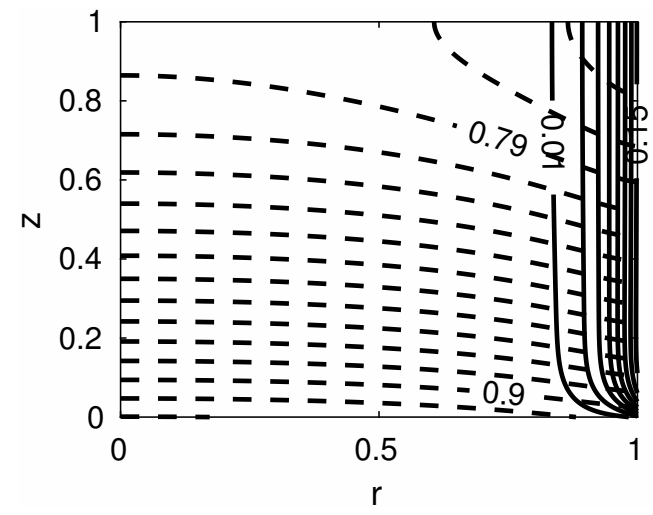

(d) $\Gamma_{c}=0.01, \Gamma_{b}=1$

Figure 7: Series of profiles showing how variation in the cell permeability coefficients $\Gamma_{c}$ and $\Gamma_{b}$ affects the spatial distribution of the chondrocyte (solid lines) and bone cell (broken lines) concentrations at $t=1$ for hydrogels that are initially devoid of cells $\left(c_{0}=b_{0}=0\right)$. With the exception of $\Gamma_{c}$ and $\Gamma_{b}$ all parameter values are held fixed at the values stated in Table 1. (a) $\Gamma_{c}=0.001$ and $\Gamma_{b}=0.1$, (b) $\Gamma_{c}=1, \Gamma_{b}=0.1$, and the chondrocyte contours are at 0.1 intervals, (c) $\Gamma_{c}=0.01$ and $\Gamma_{b}=0.02$, (d) $\Gamma_{c}=0.01$ and $\Gamma_{b}=1$. 


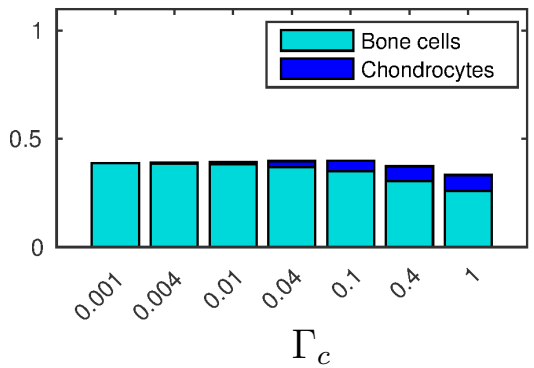

(a)

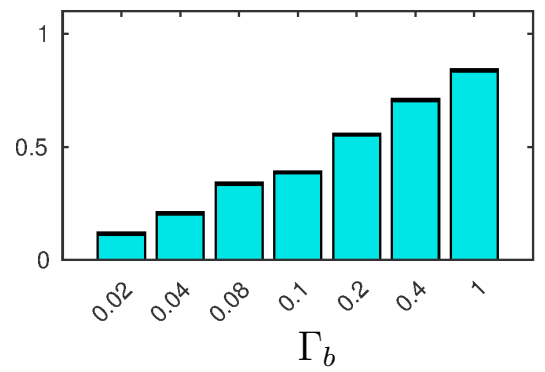

(b)

Figure 8: Series of bar plots showing how the bone cell and chondrocyte content of the hydrogel at $t=1$ changes as the interface permeabilities $\Gamma_{c}$ and $\Gamma_{b}$ are varied. These simulations were computed for an initially unseeded hydrogel. Parameter values: as per Figure 7, except that in panel (a) $\Gamma_{b}=0.1$ and in panel (b) $\Gamma_{c}=0.01$.

ing to 28 days) change as $\Gamma_{c}$ and $\Gamma_{b}$ vary. We see that increasing $\Gamma_{c}$ increases the chondrocyte content and reduces the bone cell content, whereas increasing $\Gamma_{b}$ increases the bone cell content, with negligible effect on the chondrocytes. Variations in $\Gamma_{b}$ are important to consider as there is considerable inter-species variation in ex vivo osteochondral explants. For example the subchondral plates of equine samples are thought to be impermeable to cells, whereas those of porcine samples are known to be permeable to cells (see Figure 1).

Parameter sensitivity analyses involving the diffusion coefficients $D_{c}, D_{b}$, and the parameters $\alpha_{c}$ and $\alpha_{b}$, were also performed. Larger values of $D_{c}, D_{b}$ and smaller values of $\alpha_{c}, \alpha_{b}$ (where $\alpha_{c}, \alpha_{b}$ represent the degree of inhibition that the total cell population has on the migration of both cell populations) create flatter cell distributions that are more spatially uniform as would be expected from the form of the diffusion term (results not shown). For plau- 
sible values of the diffusion coefficients the chondrocytes remain confined close to the hydrogel-cartilage interface. In summary the simulations in this section have shown that for reasonable parameter values the chondrocyte population does not generate structures that are layered in the $z$ direction.

\subsection{Zonal enhancement of proliferation and migration}

Having investigated the model with spatially uniform proliferation and migration rates and spatially uniform boundary conditions, we now consider how zonal depth variation of the chondrocyte concentration might be achieved within an initially unseeded hydrogel. In healthy cartilage the chondrocyte concentration at the cartilage surface is approximately two to three times higher than chondrocyte concentrations at the cartilage-bone interface $[2,3,4]$. Replacing our constant cartilage concentration, $C$, in the boundary condition (17), with a spatially varying chondrocyte concentration may yield a more biologically realistic depth variation in the chondrocyte population within the hydrogel. We consider a simple linear function of the form

$$
C=\frac{1+1.5 z}{1.75}
$$

where the denominator was chosen to ensure that $\int_{0}^{L} C \mathrm{~d} z=1$ when $L=$ 1. This normalisation was chosen to facilitate comparison with our earlier results where $C(z) \equiv 1$ for $0<z<L$. We assume further that we have manufactured a two-layered hydrogel and seeded the top layer with a growth factor such as TGF- $\beta$ that enhances chondrocyte proliferation and migration in the top layer of the hydrogel. We model these effects by making $\mu_{c}$ and 
$D_{c}$ spatially dependent, specifically

$$
\begin{gathered}
\mu_{c}=\mu_{c}(z)=\mu_{c 1}+\frac{\mu_{c 2}-\mu_{c 1}}{2}\left(1+\tanh \left(\frac{z-0.5}{0.01}\right)\right), \\
D_{c}=D_{c}(z)=D_{c 1}+\frac{D_{c 2}-D_{c 1}}{2}\left(1+\tanh \left(\frac{z-0.5}{0.01}\right)\right) .
\end{gathered}
$$

(The tanh function is used to smooth the interface between the upper and lower hydrogel layers). Under these assumptions, $\mu_{c} \approx \mu_{c 2}$ and $D_{c} \approx D_{c 2}$ in the top layer of the hydrogel and $\mu_{c} \approx \mu_{c 1}$ and $D_{c} \approx D_{c 1}$ in the lower region. In Figure 9 we present numerical results from a simulation in which the proliferation rate and diffusion coefficient in the upper layer of the hydrogel are ten times larger than the proliferation rate and diffusion coefficient in the lower layer. In Figure 9(a) we see that ten fold increases in $\mu_{c}$ and $D_{c}$, which give values that are much larger than estimates from the literature, enhances chondrocyte proliferation and migration, and results in a significant increase in chondrocyte levels in the upper layer. Figure 9(a) also shows that the lower proliferation rates and diffusion in the lower layer prevent the chondrocytes from colonising the lower level of the hydrogel as effectively. We note from Figure 9(b) that although the resulting structure is non-uniform in the $z$ direction, this layering is due almost entirely to the bone cells. Furthermore, there is a sharp variation in the number of cells at the interface between the upper and lower layers.

\subsection{Seeding strategies}

The failure of spatial variation in the boundary conditions, and significant increases in chondrocyte proliferation and migration, to stimulate chondrocyte infiltration through the hydrogel leads us now to consider seeding the 


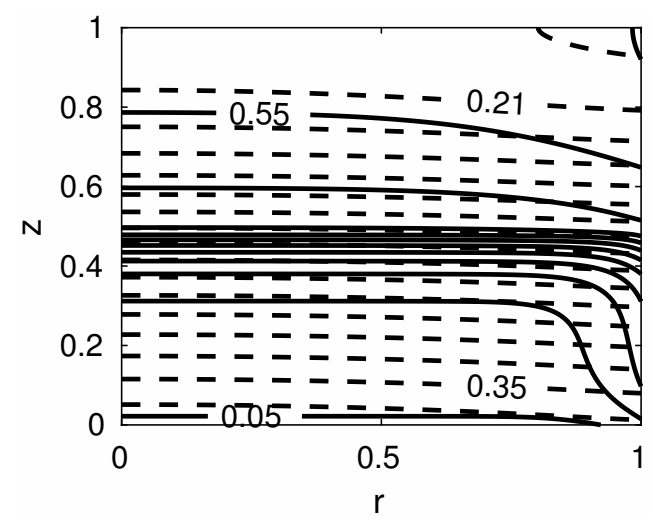

(a)

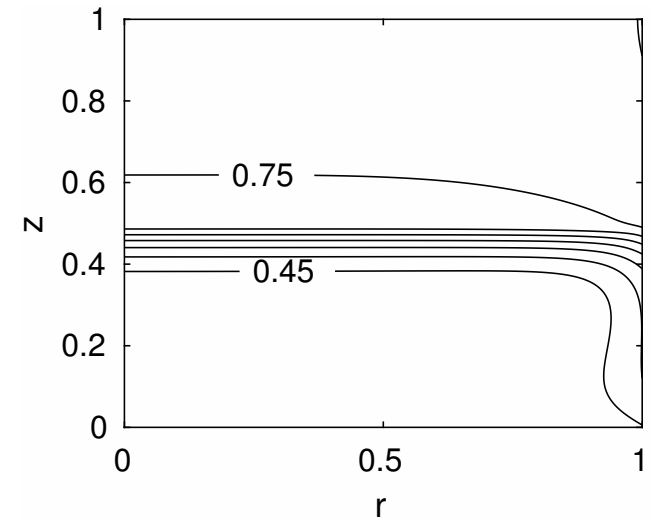

(b)

Figure 9: A numerical solution at $t=1$ for an initially unseeded hydrogel $\left(c_{0}=0\right.$, $\left.b_{0}=0\right)$. We allow cartilage permeability $C$, and proliferation and diffusion coefficients to vary spatially with depth; these quantities are given by Eqs. (19)-(21), with $D_{c 1}=0.008$, $D_{c 2}=0.08, \mu_{c 1}=0.1$ and $\mu_{c 2}=1$. The remaining parameter values are as stated in Table 1. In (a) we plot separately the chondrocyte concentrations (solid lines) at 0.05 intervals and the bone cell concentrations (broken lines). In (b) we plot the combined cell concentration $(b+c)$ at 0.05 intervals.

hydrogel with chondrocytes prior to culture. Our continued interest in generating tissue constructs with zonal architecture and chondrocyte distributions motivates us to compare uniform seeding of the hydrogel, $c_{0}(z)=0.45$, and zonal seeding,

$$
c_{0}(z)=0.45+0.15 \tanh \left(\frac{z-0.5}{0.01}\right) .
$$

We remark that the average number of chondrocytes associated with the two seeding strategies are identical. We also investigate the combined effects of non-uniform seeding strategy and zonal enhancement of the chondrocyte proliferation rate. The numerical results are presented in Figure 10. They reveal 
that the combination of a uniform proliferation rate and uniform chondrocyte seeding generates a fairly uniform chondrocyte distribution within the construct, with the cells present at levels that are almost everywhere slightly larger than those associated with the initial seeding concentration (see Figure 10(a)). Towards the hydrogel-bone interface the chondrocyte concentration dips as cells migrate from the hydrogel to the bone. The highest chondrocyte concentrations are seen along the hydrogel-cartilage interface, near to the top of the hydrogel, where $C$ is greatest. We observe slightly more depth variation in the chondrocyte concentration in Figure 10(b) when considering uniform seeding of the hydrogel and enhanced proliferation in the top layer of the hydrogel. In contrast, both the zonally seeded simulations in Figures 10(c) and 10(d) show more closely grouped chondrocyte contours around $z=0.5$ identifying a more pronounced zonal structure to the chondrocyte population, with a high density of chondrocytes in the upper layer and a low density of chondrocytes in the lower layer. In all four simulations, bone cell concentrations are lower than those seen in earlier simulations generated using the default parameter values (compare Figures 3(d) and 10). The observed reduction in bone cell levels is due to reduced bone cell proliferation and reduced bone cell migration, both effects being attributable to competition for space.

\subsection{Time-varying boundary conditions}

Thus far we have assumed that the concentrations of chondrocytes and bone cells in the tissue surrounding the hydrogel are constant. In practice it is likely that introduction of the hydrogel will alter these cell levels. Therefore we now investigate the effect of setting the the cell concentrations in 


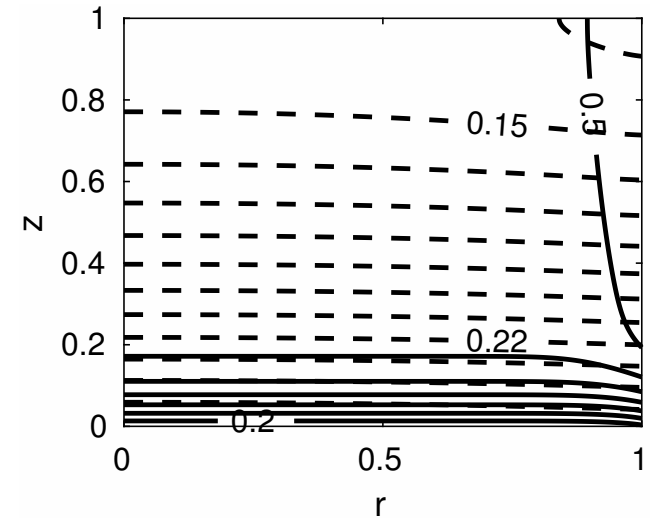

(a)

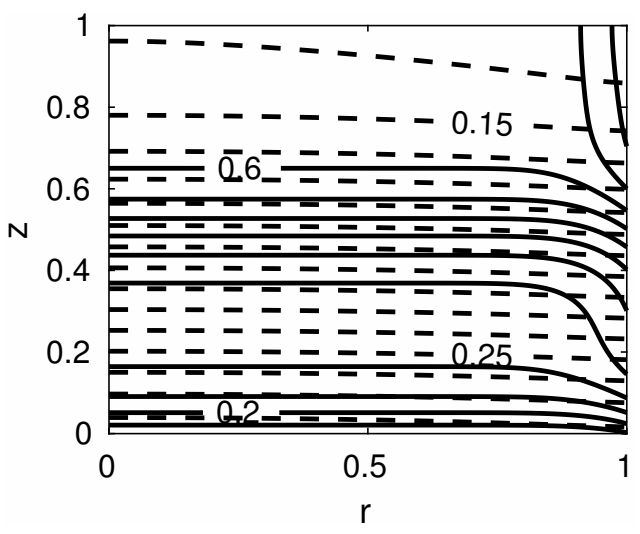

(c)

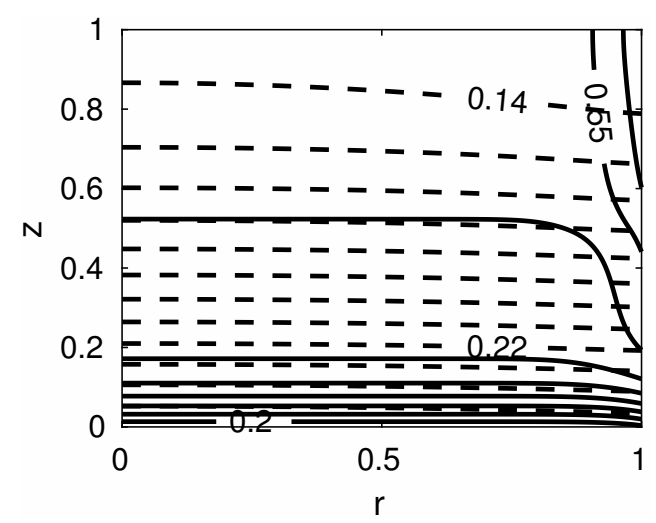

(b)

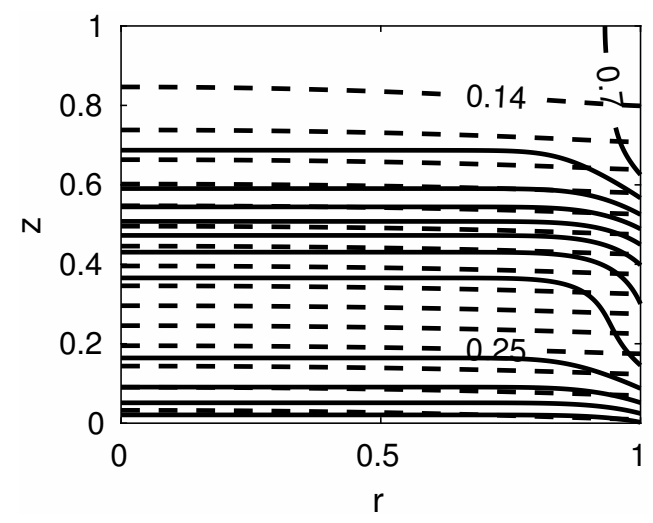

(d)

Figure 10: A series of contour plots at $t=1$ for chondrocyte concentration (solid lines at 0.05 intervals) and bone cell concentration (broken lines) showing how zonal seeding and zonal enhancement of chondrocyte proliferation affect the cellular composition of the hydrogel. In (a) there is uniform seeding $\left(c_{0}=0.45\right)$ and uniform proliferation $\left(\mu_{c}=0.1\right)$. In (b) there is uniform seeding $\left(c_{0}=0.45\right)$ and zonal proliferation enhancement $\left(\mu_{c 1}=0.1\right.$ and $\mu_{c 2}=0.3$ in Eq. (20)). In (c) there is zonal seeding (see Eq. (22)) and uniform proliferation $\left(\mu_{c}=0.1\right)$. In (d) there is zonal seeding (see Eq. (22)) and zonal enhancement of proliferation $\left(\mu_{c 1}=0.1\right.$ and $\mu_{c 2}=0.3$ in Eq. (20)). In all four simulations $C$ is defined by Eq. ((19)) and the remaining parameter values are stated in Table 1. 


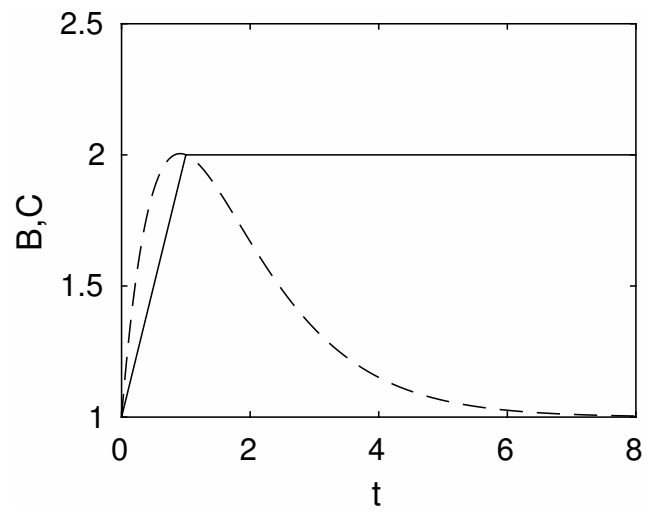

Figure 11: Sketches of functional forms used to model time variation in the quantities $B(t)$ and $C(t)$ that appear in the boundary conditions, Eqs. (17) and (18). The solid line shows $B(t)=C(t)$ defined by Eq. (23); the broken line shows $B(t)=C(t)$ defined by Eq. (24).

surrounding tissue, i.e. the quantities $B$ and $C$ that appear in Eqs. (17) and (18), to be functions of time. We begin by allowing both $B$ and $C$ to increase linearly with time until they reach a critical value and prescribe

$$
B(t)=C(t)= \begin{cases}1+t, & 0<t<1, \\ 2, & t>1,\end{cases}
$$

as illustrated by the solid line in Figure 11. The time evolution of the chondrocyte and bone concentrations arising from this choice of $B(t)$ and $C(t)$ are illustrated at in Figure 12. We observe similar features to those presented in earlier sections of this study; over time the density of both chondrocytes and bone cells increase, and - even for larger values of $B$ and $C$, and for a longer period of simulated time - the chondrocytes do not fully colonise the hydrogel.

We proceed to investigate functional forms for $B(t)$ and $C(t)$ that are characterised by an initial increase in these quantities, followed by a decrease. 


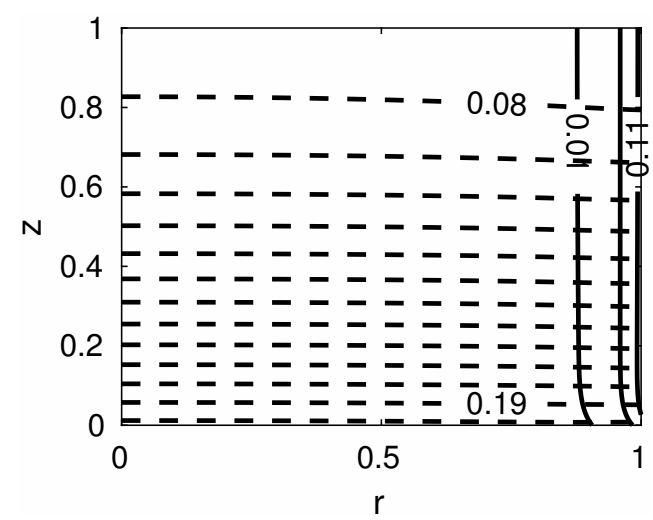

(a) $t=0.5$

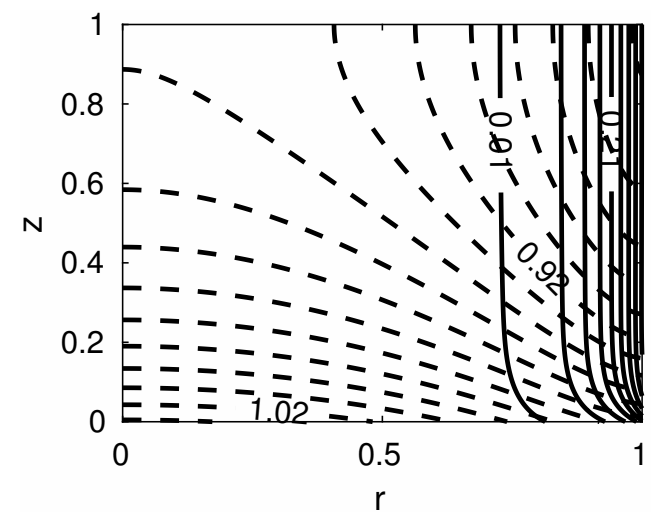

(c) $t=2$

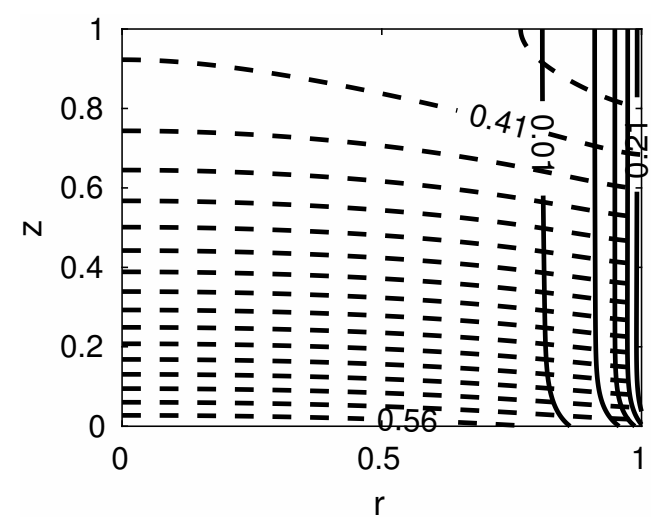

(b) $t=1$

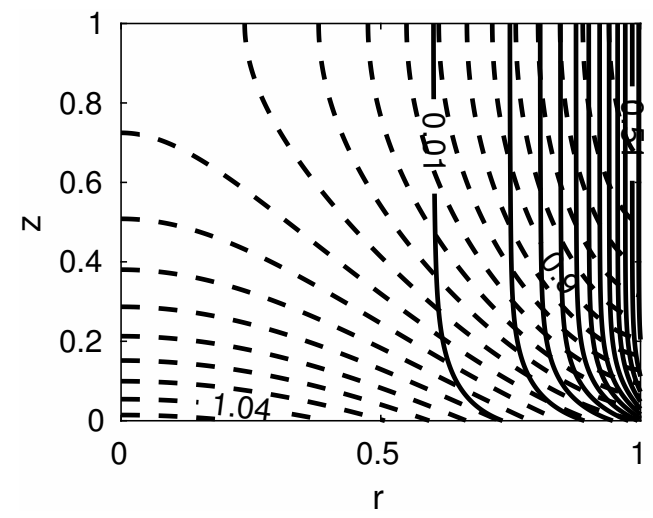

(d) $t=4$

Figure 12: Series of plots showing how the distribution of chondrocytes (solid line) and bone cells (broken lines) within a hydrogel that is initially devoid of cells $\left(c_{0}=b_{0}=0\right)$ change over time when $B(t)$ and $C(t)$ (the cell concentrations in surrounding tissue) are given by Eq. (23). The chondrocyte contours are plotted at intervals of 0.05 . (a) shows the concentrations at $t=0.5$; (b) shows the concentrations at $t=1$; (c) shows the concentrations at $t=2$; and (d) shows the concentrations at $t=4$. With the exception of $B(t)$ and $C(t)$ all other parameter values are fixed at the values stated in Table 1. 
This is representative of a wound healing response. In this case we set

$$
B(t)=C(t)=1+15\left(\mathrm{e}^{-t}-\mathrm{e}^{-1.2 t}\right) .
$$

Both $B(t)$ and $C(t)$ are illustrated by the broken line in Figure 11. The chondrocyte and bone concentrations are illustrated in Figure 13 and exhibit similar features to those seen in Figure 12. One additional point to note is that, even at $t=10$ (corresponding to 280 days) the chondrocytes do not fully colonise the hydrogel.

\section{Conclusions}

We have developed a mathematical model to study factors affecting cell migration and proliferation in hydrogels cultured in ex vivo osteochondral explants. The mathematical model has been useful in interpreting preliminary experimental data. Based on our parameter estimates and the simulations of an unseeded hydrogel with spatially uniform proliferation and diffusion coefficients we conclude that the population of cells observed in Figure 1 is likely to contain very few chondrocytes and is predominantly, if not entirely, composed of cells that have migrated from the bone. Since simulations with very small $\Gamma_{b}$ do not lead to significant increases in chondrocyte concentration, we conclude further that the presence of bone cells is not the key factor inhibiting chondrocyte infiltration. Additional model simulations indicate that even substantial enhancements to the proliferation and migration rates of chondrocytes will be insufficient to achieve good colonisation of the hydrogel and that seeding the hydrogel with chondrocytes prior to culture could achieve a more biologically realistic, layered construct. Indeed, our model simulations 


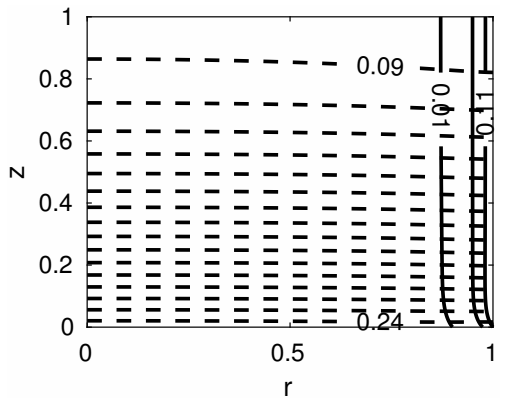

(a) $t=0.5$

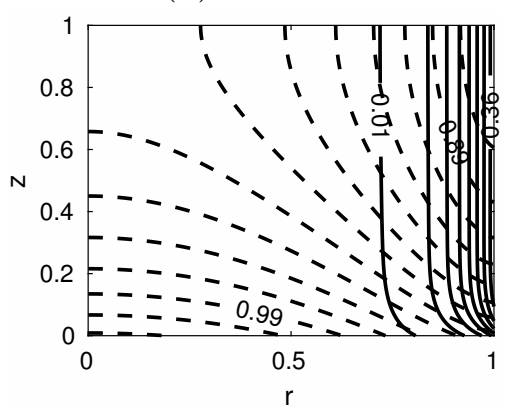

(c) $t=2$

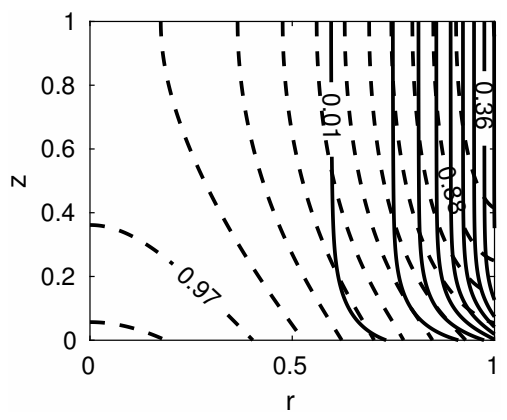

(e) $t=4$

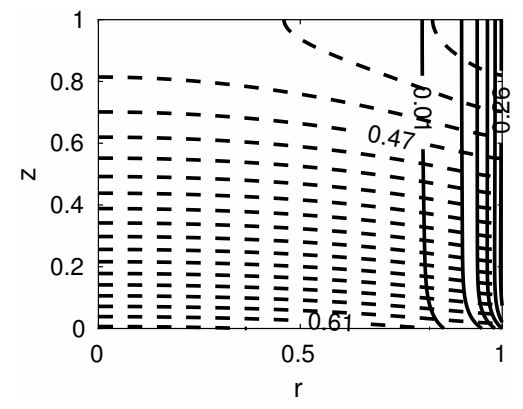

(b) $t=1$

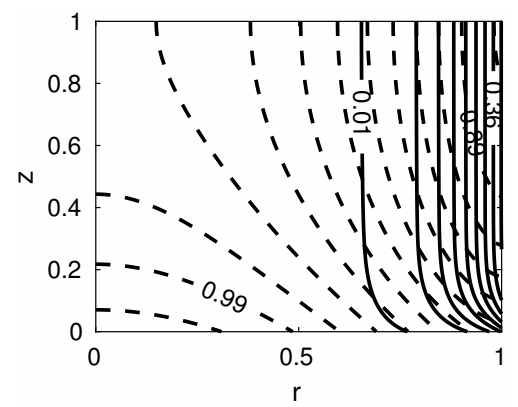

(d) $t=3$

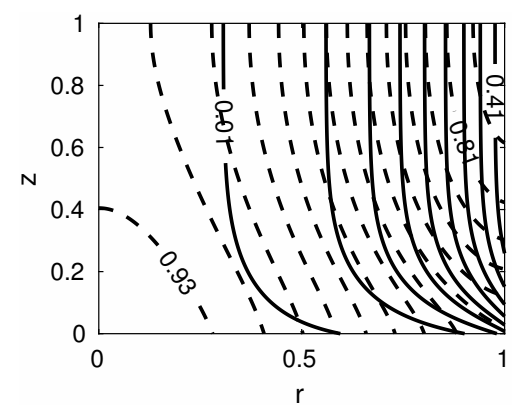

(f) $t=10$

Figure 13: Series of plots showing how the distribution of chondrocytes (solid line) and bone cells (broken lines) within a hydrogel that is initially devoid of cells $\left(c_{0}=b_{0}=0\right)$ change over time when $B(t)$ and $C(t)$ (the cell concentrations in surrounding tissue) are given by Eq. (24). The chondrocyte contours are plotted at intervals of 0.05 . (a) shows the concentrations at $t=0.5$; (b) shows the concentrations at $t=1$; (c) shows the concentrations at $t=2$; and (d) shows the concentrations at $t=4$. With the exception of $B(t)$ and $C(t)$ all other parameter values are fixed at the values stated in Table 1. 
reveal that both zonal seeding of the hydrogel and zonally enhanced chondrocyte proliferation represent promising strategies for engineering a layered distribution of chondrocytes.

Further experimental validation is needed to render the model capable of quantitative prediction: accurate estimates of cell migration and proliferation rates are needed for specific hydrogels and growth factors. There are also many ways in which the model could be extended. It could be used to investigate more detailed interactions between different cell types. For example, the cells that migrate in from the bone could be mesenchymal stem cells that differentiate into chondrocytes in the presence of chondrocytes or chondrocyte-derived growth factors [26]. Even so, we believe that the work presented here is a useful step towards a better understanding of culturing in the ex vivo osteochondral explant model. It demonstrates how mathematical modelling can be used to aid the interpretation of experimental work and guide future experimental choices resulting in more efficient progress.

\section{Acknowledgements}

The research leading to these results has received funding from the European Union Seventh Framework Programme (FP7/2007-2013) under grant agreement no. 309962 (HydroZONES). The authors thank Vivian Mouser (University Medical Center, Utrecht, Netherlands) and Linda Kock (LifeTec Group, Netherlands) for helpful discussions. 


\section{Bibliography}

[1] E. A. Makris, A. H. Gomoll, K. N. Malizos, J. C. Hu, K. A. Athanasiou, Repair and tissue engineering techniques for articular cartilage, Nature Reviews Rheumatology 11 (1) (2015) 21-34.

[2] K. D. Jadin, B. L. Wong, W. C. Bae, K. W. Li, A. K. Williamson, B. L. Schumacher, J. H. Price, R. L. Sah, Depth-varying density and organization of chondrocytes in immature and mature bovine articular cartilage assessed by 3D imaging and analysis, Journal of Histochemistry \& Cytochemistry 53 (9) (2005) 1109-1119.

[3] J. Malda, K. Benders, T. Klein, J. De Grauw, M. Kik, D. Hutmacher, D. Saris, P. van Weeren, W. Dhert, Comparative study of depthdependent characteristics of equine and human osteochondral tissue from the medial and lateral femoral condyles, Osteoarthritis and Cartilage 20 (10) (2012) 1147-1151.

[4] E. Hunziker, T. Quinn, H.-J. Häuselmann, Quantitative structural organization of normal adult human articular cartilage, Osteoarthritis and Cartilage 10 (7) (2002) 564-572.

[5] W. Schuurman, T. Klein, W. Dhert, P. Weeren, D. Hutmacher, J. Malda, Cartilage regeneration using zonal chondrocyte subpopulations: a promising approach or an overcomplicated strategy?, Journal of Tissue Engineering and Regenerative Medicine 9 (6) (2015) 669-678.

[6] J. E. Lee, K. E. Kim, I. C. Kwon, H. J. Ahn, S.-H. Lee, H. Cho, H. J. Kim, S. C. Seong, M. C. Lee, Effects of the controlled-released 
TGF- $\beta 1$ from chitosan microspheres on chondrocytes cultured in a collagen/chitosan/glycosaminoglycan scaffold, Biomaterials 25 (18) (2004) 4163-4173.

[7] T. J. Klein, J. Malda, R. L. Sah, D. W. Hutmacher, Tissue engineering of articular cartilage with biomimetic zones, Tissue Engineering Part B: Reviews 15 (2) (2009) 143-157.

[8] C. Madeira, A. Santhagunam, J. B. Salgueiro, J. M. Cabral, Advanced cell therapies for articular cartilage regeneration, Trends in Biotechnology 33 (1) (2015) 35-42.

[9] C. H. Lee, J. L. Cook, A. Mendelson, E. K. Moioli, H. Yao, J. J. Mao, Regeneration of the articular surface of the rabbit synovial joint by cell homing: a proof of concept study, The Lancet 376 (9739) (2010) 440448.

[10] B. V. Sridhar, N. R. Doyle, M. A. Randolph, K. S. Anseth, Covalently tethered TGF- $\beta 1$ with encapsulated chondrocytes in a peg hydrogel system enhances extracellular matrix production, Journal of Biomedical Materials Research Part A 102 (12) (2014) 4464-4472.

[11] A. Barbero, S. Grogan, D. Schäfer, M. Heberer, P. Mainil-Varlet, I. Martin, Age related changes in human articular chondrocyte yield, proliferation and post-expansion chondrogenic capacity, Osteoarthritis and Cartilage 12 (6) (2004) 476-484.

[12] T. I. Morales, Chondrocyte moves: clever strategies?, Osteoarthritis and Cartilage 15 (8) (2007) 861-871. 
[13] N. Hopper, F. Henson, R. Brooks, E. Ali, N. Rushton, J. Wardale, Peripheral blood derived mononuclear cells enhance osteoarthritic human chondrocyte migration, Arthritis Research \& Therapy 17 (1) (2015) 110.

[14] H. Minehara, K. Urabe, K. Naruse, A. T. Mehlhorn, K. Uchida, N. P. Südkamp, M. Itoman, A new technique for seeding chondrocytes onto solvent-preserved human meniscus using the chemokinetic effect of recombinant human bone morphogenetic protein-2, Cell and Tissue Banking 12 (3) (2011) 199-207.

[15] Y.-H. Tsai, C.-W. Chen, W.-F. T. Lai, J.-R. Tang, W.-P. Deng, S.-D. Yeh, A. Chung, C. S. Zuo, J. F. Bowley, Phenotypic changes in proliferation, differentiation, and migration of chondrocytes: 3D in vitro models for joint wound healing, Journal of Biomedical Materials Research Part A 92 (3) (2010) 1115-1122.

[16] Y. Kirilak, N. J. Pavlos, C. R. Willers, R. Han, H. Feng, J. Xu, N. Asokananthan, G. A. Stewart, P. Henry, D. Wood, M. H. Zheng, Fibrin sealant promotes migration and proliferation of human articular chondrocytes: possible involvement of thrombin and protease-activated receptors, International Journal of Molecular Medicine 17 (4) (2006) $551-558$.

[17] J. A. Sherratt, J. Murray, Models of epidermal wound healing, Proceedings of the Royal Society of London B: Biological Sciences 241 (1300) (1990) 29-36. 
[18] J. A. Sherratt, M. A. Chaplain, A new mathematical model for avascular tumour growth, Journal of Mathematical Biology 43 (4) (2001) 291-312.

[19] M. Lutianov, S. Naire, S. Roberts, J.-H. Kuiper, A mathematical model of cartilage regeneration after cell therapy, Journal of Theoretical Biology 289 (2011) 136-150.

[20] L. Edelstein-Keshet, Mathematical Models in Biology, SIAM, 1988.

[21] A. Fowler, Mathematical Models in the Applied Sciences, Cambridge University Press, Cambride, UK, 1997.

[22] N. Amor, L. Geris, J. Vander Sloten, H. Van Oosterwyck, Computational modelling of biomaterial surface interactions with blood platelets and osteoblastic cells for the prediction of contact osteogenesis, Acta Biomaterialia 7 (2) (2011) 779-790.

[23] C. Chung, C. Yang, C. Chen, Analysis of cell growth and diffusion in a scaffold for cartilage tissue engineering, Biotechnology and Bioengineering 94 (6) (2006) 1138-1146.

[24] C. Chung, C.-Y. Chen, The effect of cell sedimentation on measuring chondrocyte population migration using a boyden chamber, Journal of Theoretical Biology 261 (4) (2009) 610-625.

[25] J. Whiteley, Finite Element Methods, Springer, Cham, Switzerland, 2017.

[26] M. Chen, J. Whiteley, C. Please, A. Schwab, F. Ehlicke, S. Waters, H. Byrne, Inducing chondrogenesis in MSC/chondrocyte co-cultures us- 
ing exogenous TGF- $\beta$ : a mathematical model, bioRxiv.

URL http://biorxiv.org/content/early/2017/05/23/141119 